\title{
MULTIWAVELENGTH MONITORING OF THE DWARF SEYFERT 1 GALAXY NGC 4395. I. A REVERBERATION-BASED MEASUREMENT OF THE BLACK HOLE MASS
}

\author{
Bradley M. Peterson, ${ }^{1}$ Misty C. Bentz, ${ }^{1}$ Louis-Benoit Desroches, ${ }^{2}$ Alexei V. Filippenko, ${ }^{2}$ \\ Luis C. Ho, ${ }^{3}$ Shai Kaspi, ${ }^{4,5}$ Ari Laor, ${ }^{5}$ Dan MaOz, ${ }^{4}$ Edward C. Moran, ${ }^{6}$ \\ Richard W. Pogge, ${ }^{1}$ and Alice C. Quillen ${ }^{7}$ \\ Received 2005 April 28; accepted 2005 June 26
}

\begin{abstract}
A reverberation-mapping program on NGC 4395, the least luminous known Seyfert 1 galaxy, undertaken with the Space Telescope Imaging Spectrograph on the Hubble Space Telescope yields a measurement of the mass of the central black hole $M_{\mathrm{BH}}=(3.6 \pm 1.1) \times 10^{5} M_{\odot}$. The observations consist of two visits offive orbits each, in 2004 April and July. During each of these visits, the UV continuum varied by at least $10 \%$ (rms), and only C iv $\lambda 1549$ showed corresponding variations large enough to reliably determine the emission-line lag, which was measured to be of order $1 \mathrm{hr}$ for both visits. The size of the $\mathrm{C}$ IV-emitting region is about a factor of 3 smaller than expected if the slope of the broad-line region radius-luminosity relationship is identical to that for the $\mathrm{H} \beta$ emission line. NGC 4395 is underluminous even for its small black hole mass; the Eddington ratio of $\sim 1.2 \times 10^{-3}$ is lower than that of any other active galactic nucleus for which a black hole mass measurement has been made by emission-line reverberation.
\end{abstract}

Subject headings: galaxies: active — galaxies: nuclei — galaxies: Seyfert — quasars: emission lines — ultraviolet: galaxies

Online material: machine-readable tables

\section{INTRODUCTION}

The dwarf active galactic nucleus (AGN) in the galaxy NGC 4395 is the least luminous known object of its class (Filippenko \& Sargent 1989). While it is more than a factor of 100 less luminous than any of Seyfert's (1943) original sources, it is nevertheless a bona fide type $1 \mathrm{AGN}$; the dominant features of its ultraviolet (UV) and optical spectrum are broad emission lines, it is a hard-spectrum variable X-ray point source (Lira et al. 1999; Moran et al. 1999, 2005; Iwasawa et al. 2000; Ho et al. 2001; Shih et al. 2003), and it is a compact, high brightness temperature radio source (Moran et al. 1999; Ho \& Ulvestad 2001; Wrobel et al. 2001), although it is formally a "radio quiet" AGN (see Ho \& Peng 2001). Its spectral energy distribution from $\mathrm{X}$-ray to radio wavelengths is quite similar to those of normal type 1 AGNs (Moran et al. 1999). A surprising feature of this source is that it is found in an essentially bulgeless (Sd) galaxy (Filippenko \& Sargent 1989; Filippenko et al. 1993); both active and quiescent black holes are more likely to be found in earlytype galaxies, and there is a strong correlation between bulge mass and the mass of the central black hole (e.g., Magorrian et al. 1998; Laor 1998; Marconi \& Hunt 2003; Häring \& Rix 2004).

\footnotetext{
1 Department of Astronomy, Ohio State University, 140 West 18th Avenue, Columbus, OH 43210-1173; peterson@astronomy.ohio-state.edu, bentz@ astronomy.ohio-state.edu, pogge@astronomy.ohio-state.edu.

2 Department of Astronomy, University of California, Berkeley, CA 947203411; louis@astro.berkeley.edu, alex@astro.berkeley.edu.

${ }^{3}$ Carnegie Observatories, 813 Santa Barbara Street, Pasadena, CA 91101; lho@ociw.edu.

4 Wise Observatory and School of Physics and Astronomy, Tel Aviv University, Tel Aviv 69978, Israel; shai@wise.tau.ac.il, dani@wise.tau.ac.il.

5 Department of Physics, Technion, Haifa 32000, Israel; laor@physics .technion.ac.il.

${ }^{6}$ Department of Astronomy, Wesleyan University, Van Vleck Observatory, Middletown, CT 06459; ecm@astro.wesleyan.edu.

7 Department of Physics and Astronomy, University of Rochester, Bausch and Lomb Building, Rochester, NY 14627; aquillen@pas.rochester.edu.
}

The extreme characteristics of NGC 4395 make it worthy of close scrutiny. It is an important source in several respects:

1. A number of independent indicators ( $(4.1)$ suggest that the mass of the central black hole in NGC 4395 is less than $10^{6} M_{\odot}$, thus affording us an opportunity to begin to probe the intermediate-mass region between the supermassive black holes in AGNs and massive stellar remnants found in X-ray binary systems.

2. Even if the mass of the central black hole in NGC 4395 is at the lower end of the range allowed by indirect methods (§4.1), the accretion rate in this object must be much lower than in typical Seyfert 1 galaxies and quasars, allowing us to investigate the AGN phenomenon at low accretion rates.

3. NGC 4395 provides an opportunity to extend the mass range over which the now well-known correlation between black hole mass and stellar bulge velocity dispersion, the $M_{\mathrm{BH}}-\sigma_{*}$ relationship (Ferrarese \& Merritt 2000; Gebhardt et al. 2000a; Tremaine et al. 2002), can be tested. NGC 4395 is one of an emerging class of dwarf AGNs that seem to be found primarily in bulgeless or very late type host galaxies (e.g., Greene \& Ho 2004; Barth et al. 2005), a dramatically different environment from the cores of luminous galaxies, usually giant ellipticals, which host most of the supermassive black holes whose masses have been reliably measured. We note, of course, that low-mass black holes are found in dwarf ellipticals as well (e.g., POX 52; Barth et al. 2004).

4. The low luminosity of the AGN in NGC 4395 allows us to examine correlations between luminosity and other AGN properties over a much larger range than has been done previously. Of particular interest is the correlation between AGN luminosity and size of the broad emission line region (Kaspi et al. 2000, 2005), since this relationship is widely used in estimating the masses of the putative black holes in AGNs, often requiring extrapolation above (e.g., Vestergaard 2002; McLure \& Jarvis 2002) and below (e.g., Greene \& Ho 2004) its known range of validity. 
A property of higher luminosity AGNs shared by NGC 4395 is continuum flux variability (e.g., Skelton et al. 2005). The presence of broad lines in the UV and optical spectra thus suggest that emission-line reverberation mapping (Blandford \& McKee 1982; Peterson 1993; Netzer \& Peterson 1997) might be used to determine the size of the broad-line region (BLR) in this source, as well as the mass of the central object. Extrapolation of the relationship between continuum luminosity and BLR radius of Kaspi et al. $(2000,2005)$ to the low luminosity of NGC 4395 suggests that the size of the $\mathrm{H} \beta$-emitting region in this case should be of order light-hours, more than an order of magnitude smaller than any previously measured reverberation timescale in AGNs. Archival Hubble Space Telescope (HST) spectra from the study of Filippenko et al. (1993) show that suitable continuum variability is detectable in NGC 4395 on such scales, which indicates that reverberation techniques might be successfully applied to this low-luminosity AGN.

In this paper, we describe the results of a UV reverberationmapping program that we have undertaken to determine the size of the BLR and the mass of the central object in this source. Contemporaneous X-ray and optical observations were also obtained, and these will be presented in forthcoming papers. The observations and data reduction are outlined in $\S 2$, and the data analysis and mass determination are described in $\S 3$. The results of this experiment and their implications are discussed in $\S 4$. Our conclusions are summarized in $\S 5$.

\section{OBSERVATIONS AND DATA REDUCTION}

We observed NGC 4395 with the HST Space Telescope Imaging Spectrograph (STIS) using the FUV MAMA detector with the G140L grating, which covers the spectral range 1150-1700 . All observations were made in a time-tag mode (Dashevsky et al. 2000) to allow flexibility in trading off the competing needs of time resolution and signal-to-noise ratio $(\mathrm{S} / \mathrm{N})$. On account of well-documented sensitivity to charged particles, UV observations with STIS were restricted to the five orbits per day that were unaffected by passage through the South Atlantic Anomaly.

We initially scheduled two sets of observations on consecutive days. The first session, visit 1, began on 2004 April 10 03:19 (UT dates are used throughout this paper). The second session, visit 2, began the next day, on 2004 April 11 03:43. Five orbits of observations were obtained during each visit. Unfortunately, the HST Fine Guidance Sensor was able to acquire only a single guide star for the five orbits of visit 1 , with the result that the observations were acquired under gyro control. This led to a slow drift of the target in the aperture, thereby degrading the photometric accuracy of the observation. Consequently, NGC 4395 was reobserved during a later session, visit 3,

TABLE 1

Light CuRves for Visit 2

\begin{tabular}{|c|c|c|}
\hline $\begin{array}{c}\text { Time } \\
(\mathrm{JD}-2,453,106) \\
(1)\end{array}$ & $\begin{array}{c}F_{\lambda}(1350 \AA) \\
\left(10^{-14} \operatorname{ergs~s}^{-1} \mathrm{~cm}^{-2} \AA^{-1}\right) \\
(2)\end{array}$ & $\begin{array}{c}F(\mathrm{C} \text { IV } \lambda 1549) \\
\left(10^{-12} \underset{\text { ergs s}}{-1} \mathrm{~cm}^{-2}\right) \\
(3)\end{array}$ \\
\hline $0.69405 \ldots$ & $0.801 \pm 0.044$ & $1.414 \pm 0.095$ \\
\hline 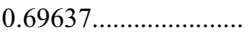 & $0.811 \pm 0.045$ & $1.272 \pm 0.085$ \\
\hline $0.69868 \ldots \ldots$ & $0.867 \pm 0.048$ & $1.243 \pm 0.083$ \\
\hline $0.70100 \ldots \ldots \ldots \ldots \ldots \ldots \ldots \ldots \ldots \ldots \ldots$ & $0.808 \pm 0.044$ & $1.324 \pm 0.089$ \\
\hline $0.70331 \ldots .$. & $0.810 \pm 0.045$ & $1.186 \pm 0.079$ \\
\hline
\end{tabular}

Note.-Table 1 is published in its entirety in the electronic edition of the Astrophysical Journal. A portion is shown here for guidance regarding its form and content.
TABLE 2

Light Curves for Visit 3

\begin{tabular}{|c|c|c|}
\hline $\begin{array}{c}\text { Time } \\
(\mathrm{JD}-2,453,190) \\
(1)\end{array}$ & $\begin{array}{c}F_{\lambda}(1350 \AA) \\
\left(10^{-14} \mathrm{ergs} \mathrm{s}^{-1} \mathrm{~cm}^{-2} \AA^{-1}\right) \\
(2)\end{array}$ & $\begin{array}{c}F(\mathrm{C} \text { IV } \lambda 1549) \\
\left(10^{-12} \mathrm{ergs} \mathrm{s}^{-1} \mathrm{~cm}^{-2}\right) \\
(3)\end{array}$ \\
\hline $0.01837 .$. & $2.780 \pm 0.075$ & $2.601 \pm 0.101$ \\
\hline $0.02069 \ldots \ldots \ldots \ldots \ldots \ldots \ldots \ldots \ldots \ldots$ & $2.831 \pm 0.076$ & $2.538 \pm 0.099$ \\
\hline $0.02300 \ldots \ldots \ldots \ldots \ldots \ldots \ldots$ & $2.916 \pm 0.079$ & $2.778 \pm 0.108$ \\
\hline 0.02532 . & $2.885 \pm 0.078$ & $2.733 \pm 0.107$ \\
\hline $0.02763 \ldots$ & $3.153 \pm 0.085$ & $2.640 \pm 0.103$ \\
\hline
\end{tabular}

Note.-Table 2 is published in its entirety in the electronic edition of the Astrophysical Journal. A portion is shown here for guidance regarding its form and content.

beginning on 2004 July 3 12:07. All observations were made through a $52^{\prime \prime} \times 0 . .5$ slit at position angles $116^{\circ} .5,119^{\circ} .7$, and $60^{\circ} .9$ for visits $1-3$, respectively.

A continuum window of width $30 \AA$ was adopted to obtain $\mathrm{S} / \mathrm{N} \approx 50$ in individual exposures of $200 \mathrm{~s}$. This resulted in nine exposures for the first orbit of each visit and 14 exposures for each of the four remaining orbits. The individual time-tagged events in each $200 \mathrm{~s}$ exposure were converted into a single accumulated image that was then calibrated using standard STIS pipeline software. In addition, we created a single average spectrum for each orbit; each of these full-orbit exposures was used to set the aperture for spectral extraction in the $200 \mathrm{~s}$ exposures of that orbit. The extraction window in the cross-dispersion direction was 12 pixels, or 0 ". 288 .

Once the spectra were extracted, we measured the mean value of the continuum flux over the range 1335-1365 $\AA$. To measure the $\mathrm{C}$ IV $\lambda 1549$ emission-line flux, we used a simple summing function with a two-point interpolation of the average continuum values between $1520-1530$ and $1570-1580 \AA$. The errors in the flux measurements were estimated conservatively by assuming that any differences between adjacent exposures are stochastic, not real, and that the fractional error in each $200 \mathrm{~s}$ exposure is the same. Specifically, we computed for each pair of observations separated by no more than $260 \mathrm{~s}$ the fractional change in flux and assumed that the variance in this quantity was twice the square of the fractional uncertainty for each point. We note in passing that we also attempted to measure the flux in the weaker emission lines Si IV $\lambda 1400+\mathrm{O}$ IV] $\lambda 1402$ and He II $\lambda 1640$; these two features clearly show up in the variable part of the spectrum $(\S 3.2)$, but their light curves are too noisy to be usable. It was also not possible to obtain a useful light curve for $\operatorname{Ly} \alpha \lambda 1215$ because its apparent variability is dominated by variations of the geocoronal Ly $\alpha$ contribution.

This procedure produced the light curves that are given in Tables 1 and 2 and shown in Figures 1 and 2 for visits 2 and 3, respectively. We also attempted to extract light curves from the visit 1 spectra, but these proved to be unusable on account of the systematic photometric errors. In Table 3 we summarize some of the variability characteristics of the visits 2 and 3 light curves in terms of the excess variance $F_{\text {var }}$ (Rodríguez-Pascual et al. 1997 ) in the light curve and the maximum-to-minimum flux ratio $R_{\max }$. Significant variations of the continuum and C IV emission-line flux are seen on timescales less than a single orbit. Larger differences are seen over the 3 month interval between the two visits: between visit 2 and visit 3 , the continuum flux increased by a factor of $\sim 3.5$ and the $C$ IV line flux increased by a factor of $\sim 2.1$. The continuum variations as characterized by the excess variance were about $38 \%$ larger during visit 2 than 


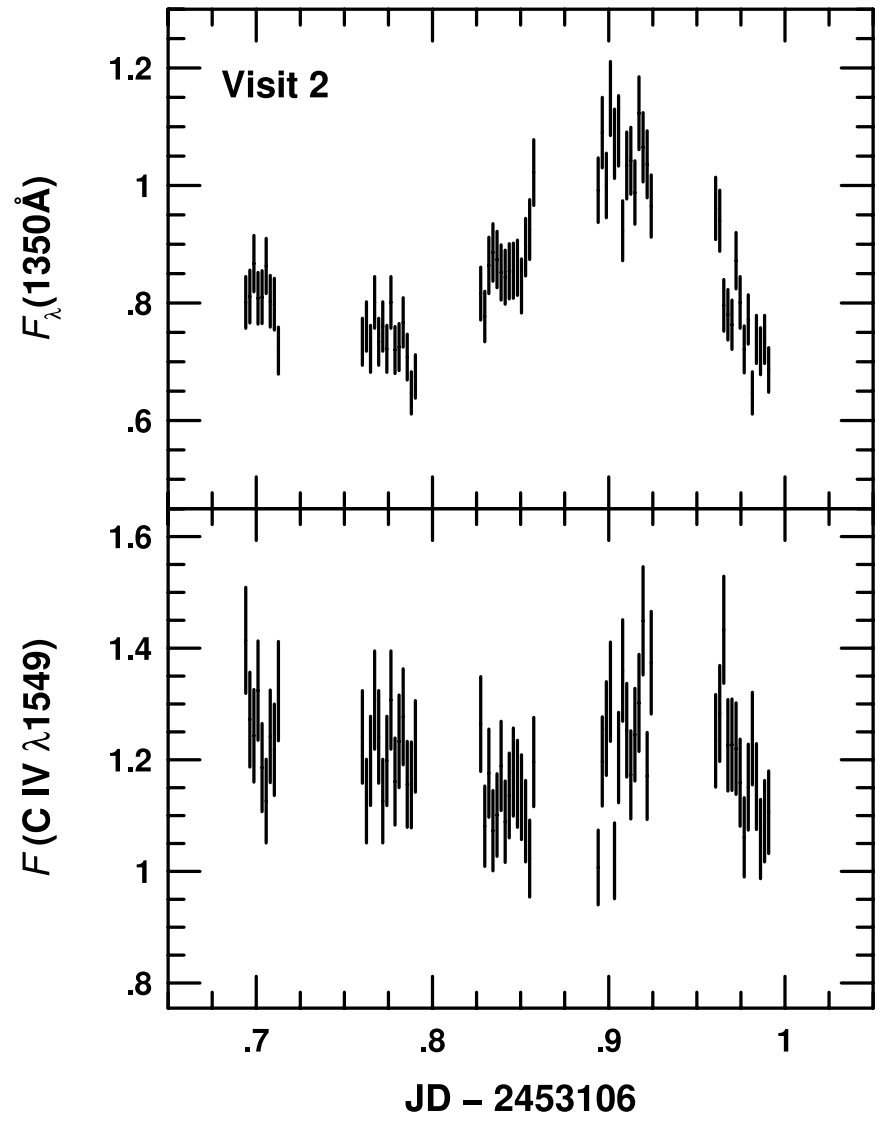

Fig. 1.-Light curves for the UV continuum at $1350 \AA$ (top) and the $\mathrm{C}$ IV $\lambda 1549$ emission line on 2004 April 11 (visit 2). The continuum flux density is in units of $10^{-14} \mathrm{ergs} \mathrm{s}^{-1} \mathrm{~cm}^{-2} \AA^{-1}$, and the emission-line flux is in units of $10^{-12} \mathrm{ergs} \mathrm{s}^{-1} \mathrm{~cm}^{-2}$.

during visit 3 , and in both visits, the continuum variations were about a factor of 3 larger than those in the $\mathrm{C}$ IV line.

\section{DATA ANALYSIS}

\subsection{Time-Series Analysis}

\subsubsection{Measuring the Emission-Line Lag}

Our goal is to measure the timescale for response of the $\mathrm{C}$ IV emission line to the continuum variations by cross-correlation of the UV continuum and $\mathrm{C}$ IV light curves. From a computational point of view, cross-correlation is effected by shifting one time series relative to the other by discrete amounts and computing the linear correlation coefficient between matched continuum/ emission-line pairs (see Peterson 2001 for a tutorial on crosscorrelation as applied to reverberation analysis). Since astronomical time series are rarely regularly sampled, one is forced to impose regularity on the time series by either (1) interpolating between real data points in the light curve to match pairs of points for any arbitrary discrete shift (e.g., Gaskell \& Sparke 1986; Gaskell \& Peterson 1987; White \& Peterson 1994; Peterson et al. 1998, 2004) or (2) binning the data, i.e., by matching points within some allowable bin width in time shift (e.g., Edelson \& Krolik 1988; Alexander 1997).

For reverberation analysis, the former, known as the interpolated cross-correlation function (ICCF), generally performs better than the latter, known as the discrete correlation function (DCF), as argued by White \& Peterson (1994). However, the particular situation we are faced with here, with large gaps in otherwise well-sampled time series on account of Earth occultation during part of each orbit, is much better suited to the

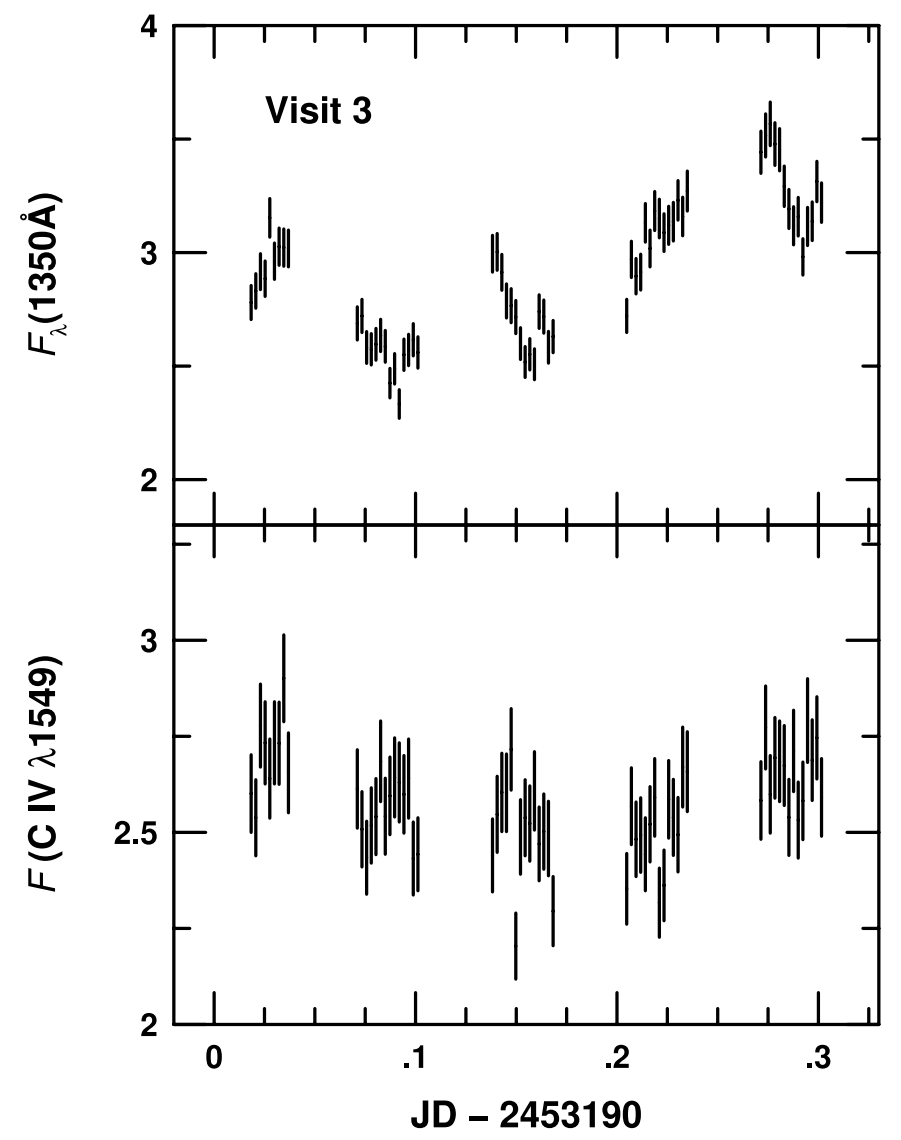

FIg. 2.- Light curves for the UV continuum at $1350 \AA$ (top) and the $\mathrm{C}$ IV $\lambda 1549$ emission line on 2004 July 3 (visit 3). Fluxes are as in Fig. 1.

DCF than the ICCF. Some experimentation with both real and simulated data revealed that the DCF can perform remarkably well, provided that (1) the emission-line lag is characterized by the centroid $\tau_{\text {cent }}$ of the DCF rather than by $\tau_{\text {peak }}$, i.e., the lag where the linear correlation coefficient has its maximum value $r_{\max }$, and that (2) poorly populated bins (i.e., lags at which there are few matched continuum/emission-line pairs) are discarded prior to calculation of the centroid. In general, it seems to be true that the emission-line lag is better characterized by $\tau_{\text {cent }}$ rather than by $\tau_{\text {peak }}$; the latter is much less stable than the former in both the DCF and ICCF, and $\tau_{\text {peak }}$ is much less stable in the DCF than in the ICCF.

With these modifications to how the DCF is usually implemented (e.g., Edelson \& Krolik 1988), we were able to employ our usual model-independent Monte Carlo method (Peterson et al. 1998, 2004) to compute the cross-correlation lag and its uncertainty. The method works as follows:

1. The continuum and emission-line light curves, consisting of $M$ and $N$ total data points, respectively, are resampled by drawing $M$ and $N$ random selections from the original time series without regard to whether a particular point has been selected previously. Thus, some points are selected multiple times and others are not selected at all (in such a Poisson process, typically a fraction $1 / e$ remains unselected).

2. The flux error associated with each selected data point $i$ is reduced by a factor $n_{i}^{1 / 2}$, where $n_{i}$ is the number of times the data point was selected in step 1 .

3. The flux value associated with each selected data point is altered by a random Gaussian deviate of width equal to the weighted error associated with that point. 
TABLE 3

VARIABILITY PARAMETERS

\begin{tabular}{|c|c|c|c|c|}
\hline $\begin{array}{c}\text { Time Series } \\
\text { (1) }\end{array}$ & $\begin{array}{l}\text { Number of Epochs } \\
\text { (2) }\end{array}$ & $\begin{array}{l}\text { Mean Flux } \\
\text { (3) }\end{array}$ & $\begin{array}{l}F_{\text {var }} \\
(4)\end{array}$ & $\begin{array}{l}R_{\max } \\
(5)\end{array}$ \\
\hline \multicolumn{5}{|l|}{ Visit 2: } \\
\hline Continuum..................... & 65 & $0.849 \pm 0.126^{\mathrm{a}}$ & 0.138 & $1.774 \pm 0.139$ \\
\hline 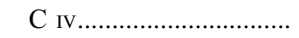 & 65 & $1.202 \pm 0.097^{\mathrm{b}}$ & 0.045 & $1.439 \pm 0.136$ \\
\hline \multicolumn{5}{|l|}{ Visit 3: } \\
\hline Continuum...................... & 65 & $2.916 \pm 0.302^{\mathrm{a}}$ & 0.100 & $1.529 \pm 0.059$ \\
\hline 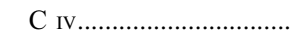 & 65 & $2.576 \pm 0.127^{b}$ & 0.029 & $1.317 \pm 0.074$ \\
\hline
\end{tabular}

${ }^{\mathrm{a}}$ In units of $10^{-14} \mathrm{ergs} \mathrm{s}^{-1} \mathrm{~cm}^{-2} \AA^{-1}$

b In units of $10^{-12} \mathrm{ergs} \mathrm{s}^{-1} \mathrm{~cm}^{-2}$.

4. The two resampled and altered time series are crosscorrelated using the DCF code, and the centroid of the resulting cross-correlation function is computed after rejecting time bins with too few data points. The centroid of the cross-correlation function is recorded for this single realization.

5. The process is repeated for a large number of realizations, and the recorded values of the centroid are used to build up a cross-correlation centroid distribution (CCCD). The mean and standard deviation of this distribution, after removing obviously failed realizations, are taken to be the emission-line lag and its associated uncertainty, respectively.

In Figure 3 we show the cross-correlation functions obtained by cross-correlating the UV continuum (measured at $1350 \AA$ ) and the $\mathrm{C}$ IV emission-line fluxes given in Tables 1 and 2 and shown

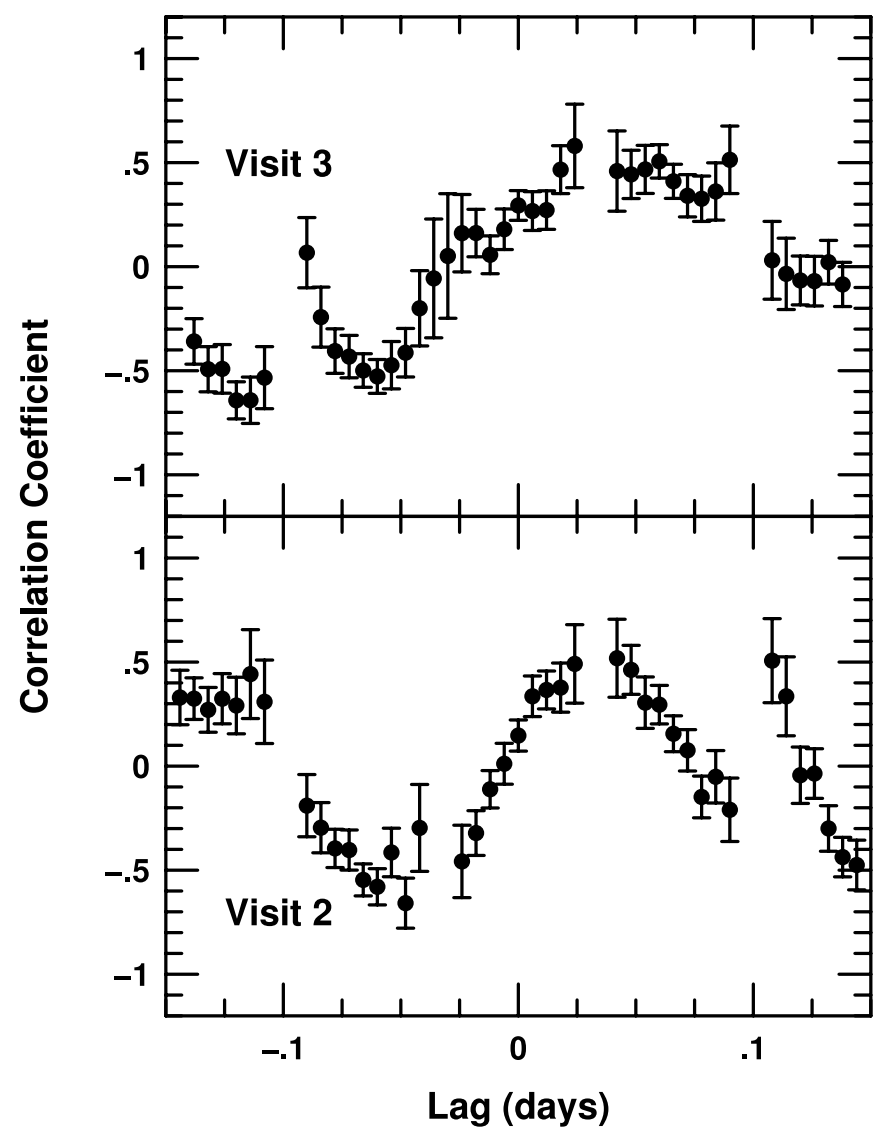

FIG. 3.-DCFs for visit 2 (bottom) and visit 3 (top), obtained by crosscorrelating the continuum and emission-line light curves shown in Figs. 1 and 2 for visits 2 and 3, respectively. Bins based on fewer than 15 points are omitted. in Figures 1 and 2. For both visits, the peak value of the crosscorrelation functions is $r_{\max } \gtrsim 0.5$. For the cross-correlations shown in Figure 3, the mean number of pairs per bin is 74 with a standard deviation of 38 . We omitted from the centroid calculation the DCF bins with fewer than 15 independent pairs of data points. We applied the Monte Carlo method described above, and with 4000 simulations produced the CCCDs that are shown in Figure 4. Based on these CCCDs, the $\mathrm{C}$ iv emission-line lags and their associated uncertainties are $\tau_{\text {cent }}=0.033_{-0.013}^{+0.017}$ days (i.e., $48_{-19}^{+24}$ minutes) for visit 2 and $\tau_{\text {cent }}=0.046_{-0.013}^{+0.017}$ days (i.e., $66_{-19}^{+24}$ minutes) for visit 3 . The quoted errors correspond to

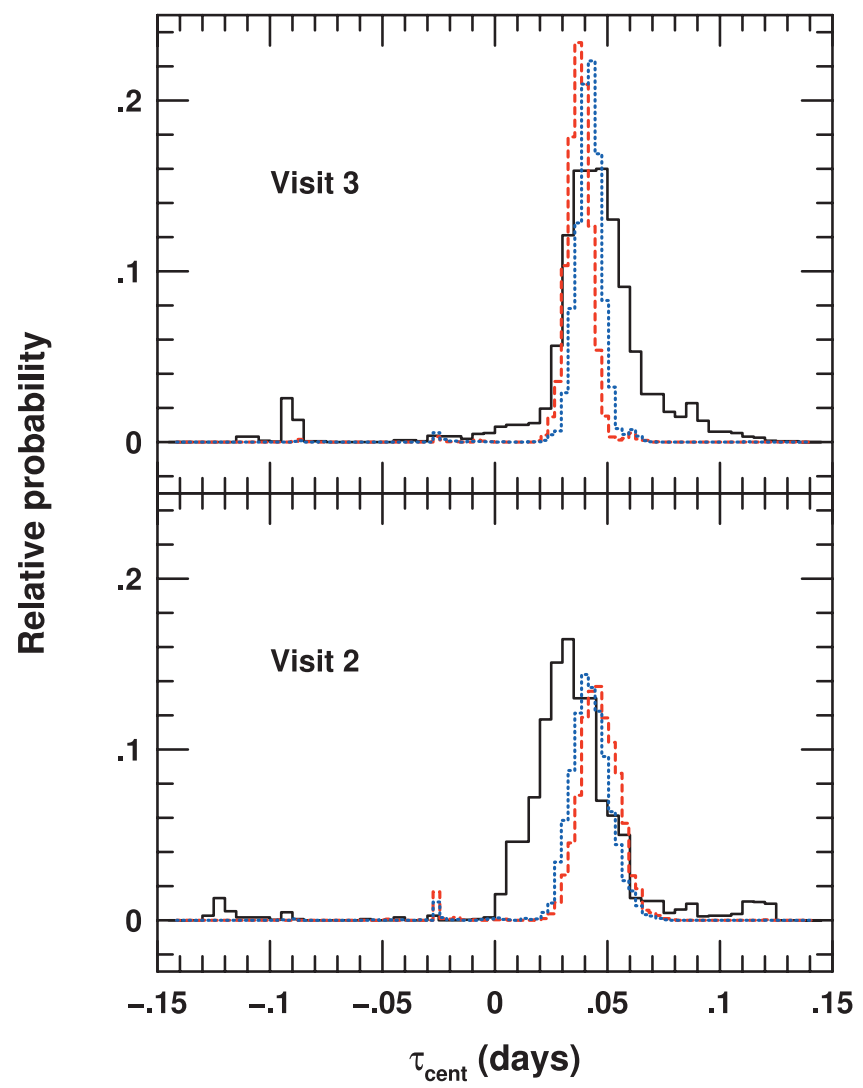

FIG. 4.-CCCDs for visit 2 (bottom) and visit 3 (top) are shown as black solid lines. The centroids of these functions are $\tau_{\text {cent }}=0.033_{-0}^{+0.017}$ days (i.e., $48_{-19}^{+24}$ minutes) for visit 2 and $\tau_{\text {cent }}=0.046_{-0.013}^{+0.017}$ days (i.e., $66_{-19}^{+24}$ minutes) for visit 3. Also shown are CCCDs from the simulations described in the text. The blue dotted lines are for thin-shell geometries of radius $R=c \tau_{\text {cent }}$, and the red dashed lines are for $i=0^{\circ}$ rings with $R=c \tau_{\text {cent. }}$. The slight offset between peaks of the thin-shell model CCCDs and the peak of the experimental CCCD is a consequence of the simple continuum interpolation model used to bridge the gaps between orbits. 


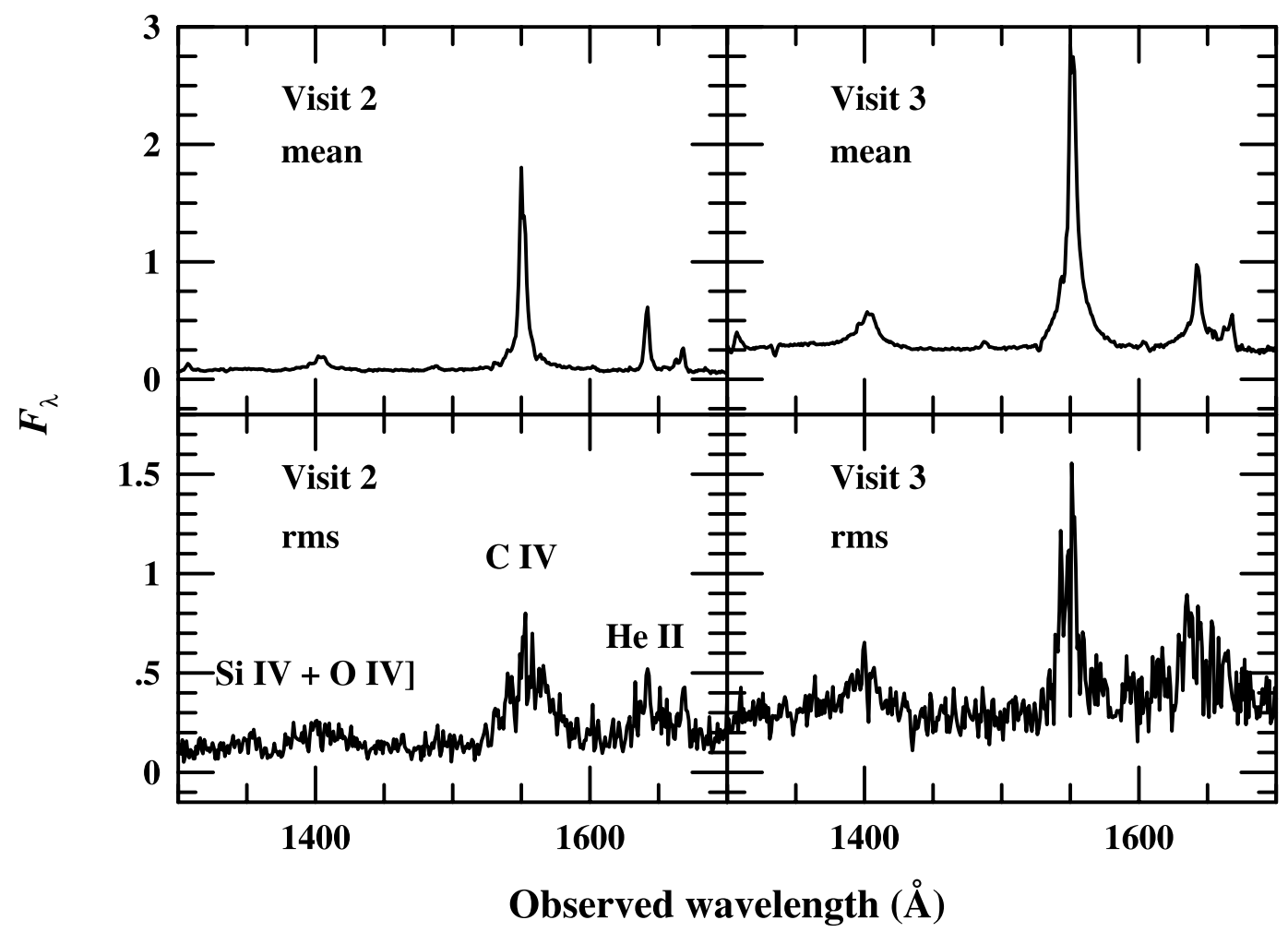

FIG. 5.-Mean (top) and rms (bottom) spectra for visit 2 (left) and visit 3 (right). Flux density is in units of $10^{-13} \mathrm{ergs} \mathrm{s}^{-1} \mathrm{~cm}^{-2} \AA^{-1}$. The rms spectrum isolates the variable part of the spectrum, and we use the line dispersion of the $\mathrm{C}$ iv line (the strongest feature in each spectrum) in the rms spectrum to characterize the velocity dispersion of the gas in the black hole mass calculation. The strongest emission lines are identified in the bottom left panel.

the conventional $68 \%$ confidence range unless otherwise noted. That these lags are different is not surprising, since the mean continuum flux is a factor of 3.4 higher for visit 3 compared to visit 2 . In the well-studied case of NGC 5548, the $\mathrm{H} \beta$ lag is found to be dependent on the mean luminosity in the same sense (Peterson et al. 2002), and it is reasonable to expect the same sort of effect in this case.

\subsubsection{Reliability of the Emission-Line Lags}

Given the unusual sampling pattern of the observations and the short duration of each visit ( $\sim 0.28$ days, or just under $7 \mathrm{hr}$ ), one can ask whether we might be misled by these results. Specifically, we wish to consider the possibility that the C IVemitting region is significantly larger than the value we obtained and that our result is spurious.

We therefore carried out Monte Carlo simulations, similar to those described by Peterson et al. (1998), in an attempt to assess how likely a spurious result might be. We first generated a model continuum light curve by fitting the observed continuum light curve with a low-order polynomial. The model light curve was then convolved with a transfer function, as described below, to produce a model emission-line curve. The model continuum and emission-line light curves were subsequently sampled in the same pattern as the real observations, and the fluxes of the sampled data points were altered by random Gaussian deviates with variances chosen to be consistent with the adopted flux uncertainties in the real data, in order to simulate the effects of noise. These simulated data were then analyzed with the same algorithms used on the real data and constituted a single realization. Multiple realizations were used to build up a distribution of measured emission-line lags to see how often particular values of the lag are produced.
For the sake of simplicity, we employed two types of transfer function:

1. A thin spherical shell of specified radius $R$.- Such a transfer function has constant response from $\tau=0$ to $2 R / c$ and is zero elsewhere.

2. $A$ thin ring of radius $R$ and inclination $i=0^{\circ}$ (i.e., $a$ face-on ring). - This transfer function is a delta function at $\tau=R / c$.

The emission-line response in both cases should lead to a centroid measurement $\tau_{\text {cent }}=R / c$ for ideal data. These two transfer functions represent extreme behavior: the response of the inclined ring is highly localized in time at $R / c$, and the thin spherical shell response is spread out evenly over the range $0 \leq \tau \leq 2 R / c$.

We first considered a model BLR with a response time $\tau_{\text {model }}$ equal to that of the measured cross-correlation centroids, i.e., $\tau_{\text {visit }}=0.033$ days for visit 2 and $\tau_{\text {visit }}=0.046$ days for visit 3 . For visit 2 , the DCF yielded lags with $r_{\max } \geq 0.5$, i.e., of significance comparable to the observed correlation, for nearly $60 \%$ of the disk simulations and for more than $70 \%$ of the shell simulations. For visit 3, more than $98 \%$ of both simulations yielded lags with $r_{\max } \geq 0.5$. The CCCDs for the successful simulations are shown in Figure 4; the model CCCDs are reasonably consistent with the observed CCCDs and quoted uncertainties, given the highly simplified transfer function models, the adopted model for the continuum variations, and the suboptimal sampling pattern of the observations. These simulations demonstrate the veracity of the method by establishing self-consistency; simulated data based on simple models with attributes inferred from the observations in most cases reproduce the measurements we obtained from the actual data. 
We then considered whether the observed lags could be obtained with a true response time that was larger than the size we infer from the observations. For a modestly larger BLR with $\tau_{\text {model }}=0.060$ days, the results were only slightly degraded from the first set of simulations in which $\tau_{\text {model }}=\tau_{\text {visit }}$. For a larger BLR with $\tau_{\text {model }}=0.120$ days, the failure rate became high; only $2 \%$ of the simulations yielded $0 \leq \tau_{\text {cent }} \leq \tau_{\text {visit }}$, except that the visit 2 simulations with the thin-shell model yielded successful DCFs (i.e., $r_{\max } \geq 0.5$, consistent with the observed result) $\sim 40 \%$ of the time, and in these cases $36 \%$ of the simulations yielded a centroid measurement in the range $0 \leq \tau_{\text {cent }} \leq$ $\tau_{\text {model }}$. In other words, in our worst case, about $15 \%$ of the realizations produced a value consistent with our observational result even though $\tau_{\text {model }}$ was significantly larger than $\tau_{\text {cent }}$.

These simulations, especially the difference between the thin-shell simulations for visits 2 and 3, show that the crosscorrelation results have some sensitivity to the pattern of continuum variability and the sampling pattern of the observations, as is well known from other simulations. However, the fact that visit 2 and visit 3 are two completely independent sets dramatically improves our confidence in our lag measurements: while a larger BLR cannot be excluded at high confidence based on the visit 2 data, $\tau_{\text {model }} \gtrsim 0.12$ days can be excluded at $98 \%$ confidence from the visit 3 data. In summary, these simulations reassure us that our lag measurements are probably not spurious.

\subsection{Line Width Measurement and Mass Determination}

As described by Peterson et al. (2004), we characterize the width of the variable part of the emission line by measuring both the line dispersion $\sigma_{\text {line }}$ (i.e., the second moment of the line flux) and the full-width at half maximum (FWHM) in the rootmean-square (rms) spectrum formed by combining all of the individual spectra. In this case, the individual $200 \mathrm{~s}$ integrations have adequate $\mathrm{S} / \mathrm{N}$ for measurement of continuum and integrated emission-line fluxes, but the $\mathrm{S} / \mathrm{N}$ per pixel is still quite low. To avoid producing rms spectra that are dominated by photon noise, we combined all the spectra into five orbital averages for each visit. The mean and rms spectra were then constructed from these orbital averages, and they are shown in Figure 5 for visits 2 and 3 .

In order to assess the uncertainties in these measures, we employed a Monte Carlo method described by Peterson et al. (2004) that is similar to that used in the time-series analysis. Specifically, for a single realization we make $N$ random selections of $N$ spectra, without regard to whether any particular spectrum has already been selected. The mean and line dispersion are measured in the rms spectrum formed by these $N$ selections. Multiple realizations lead to distributions in the measurements of $\sigma_{\text {line }}$ and FWHM, and we take the mean and standard deviation to be the line width measurement and its uncertainty, respectively. We also

TABLE 4

C iv $\lambda 1549$ Line Width Measurements

\begin{tabular}{|c|c|c|}
\hline $\begin{array}{l}\text { Spectrum } \\
\text { (1) }\end{array}$ & $\begin{array}{c}\text { FWHM } \\
\left(\mathrm{km} \mathrm{s}^{-1}\right) \\
(2)\end{array}$ & $\begin{array}{c}\text { Line Dispersion } \sigma_{\text {line }} \\
\left(\mathrm{km} \mathrm{s}^{-1}\right) \\
(3)\end{array}$ \\
\hline \multicolumn{3}{|l|}{ Visit 2: } \\
\hline $\begin{array}{l}\text { Mean spectrum................................................. } \\
\text { rms spectrum }\end{array}$ & $\begin{array}{l}1214 \pm 2 \\
3163 \pm 1650\end{array}$ & $\begin{array}{l}1727 \pm 78 \\
3025 \pm 201\end{array}$ \\
\hline \multicolumn{3}{|l|}{ Visit 3: } \\
\hline 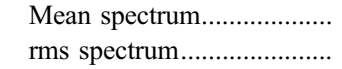 & $\begin{array}{l}1532 \pm 6 \\
2961 \pm 1166\end{array}$ & $\begin{array}{l}1662 \pm 34 \\
2859 \pm 376\end{array}$ \\
\hline
\end{tabular}

TABLE 5

Black Hole Mass Measurements

\begin{tabular}{cccc}
\hline \hline $\begin{array}{c}\text { Visit } \\
(1)\end{array}$ & $\begin{array}{c}\text { DCF Centroid } \tau_{\text {cent }} \\
(\text { days })\end{array}$ & $\begin{array}{c}\text { Line Dispersion } \sigma_{\text {line }} \\
(2)\end{array}$ & $\begin{array}{c}\text { Virial Mass } \\
\left(\mathrm{km} \mathrm{s}^{-1}\right)\end{array}$ \\
\hline $2 \ldots \ldots \ldots \ldots \ldots .$. & $(3)$ & $(4)$ \\
\hline$\ldots \ldots \ldots \ldots \ldots \ldots . .$. & $0.033_{-0.013}^{+0.017}$ & $3025 \pm 201$ & $3.2_{-1.4}^{+1.7}$ \\
\hline
\end{tabular}

note that we make a small correction for the spectrograph resolution (also as described by Peterson et al.), which we take to be FWHM $=1.2 \AA$ at C IV (Keyes 1997). Our measured line widths are given in Table 4.

This case differs rather markedly from those analyzed by Peterson et al. in that there are so few individual (i.e., only the orbital averages) spectra for these Monte Carlo simulations. Extensive tests using small numbers of spectra reveal that the line widths and uncertainties in the rms spectra are adequately determined from even a small number of spectra. The line width in the mean spectrum is also well determined, but the uncertainty in the line width is underestimated especially if the $\mathrm{S} / \mathrm{N}$ is high (as it is in this case). Our implicit assumption is that the principal source of uncertainty in the line width is simply photon statistics, although in this case, it is clear that other systematic effects that are not taken into account here, such as continuum placement and line blending, are more important. Since we do not make any additional use of the uncertainty of the line width in the mean spectrum, we will not pursue this issue further.

The mass of the putative black hole is assumed to be

$$
M_{\mathrm{BH}}=f \sigma_{\text {line }}^{2} c \tau_{\text {cent }} / G
$$

where $f$ is a scale factor that depends on the unknown kinematics and geometry of the BLR. For a sample of AGNs for which both reverberation-based masses and host-galaxy bulge velocity dispersions $\sigma_{*}$ are available, Onken et al. (2004) find empirically that $\langle f\rangle=5.5$, based on the assumption that the relationship between central black hole mass and $\sigma_{*}$, the $M_{\mathrm{BH}^{-}} \sigma_{*}$ relationship (Ferrarese \& Merritt 2000; Gebhardt et al. 2000a), is identical for quiescent and active galaxies. Adopting this value, we obtain the masses in Table 5. Note that the associated uncertainties quoted are for the random part of the uncertainty, due only to the uncertainties in $\tau_{\text {cent }}$ and $\sigma_{\text {line }}$; the scatter of the reverberation-based masses around the $M_{\mathrm{BH}^{-}} \sigma_{*}$ relationship suggests that the systematic uncertainty is approximately a factor of 3 (Onken et al. 2004). Combining the results from visits 2 and 3 yields $M_{\mathrm{BH}}=(3.6 \pm 1.1) \times 10^{5} M_{\odot}$.

\section{DISCUSSION}

\subsection{Comparison of the Black Hole Mass Measurement} with Previous Estimates

While our reverberation-based value of $M_{\mathrm{BH}}=3.6 \times 10^{5} M_{\odot}$ is the first measurement of the central mass in NGC 4395, the mass has been previously estimated by various means. Filippenko \& Ho (2003) give firm lower and upper limits on the central mass of $M>250 M_{\odot}$ from the Eddington limit and $M<6 \times 10^{6} M_{\odot}$ based on the dynamics of the central star cluster. Kraemer et al. (1999) estimate a BLR radius of 0.36 lt-days based on photoionization modeling, which combined with their quoted $\mathrm{H} \beta$ line width $\left(\mathrm{FWHM}=1500 \mathrm{~km} \mathrm{~s}^{-1}\right.$ ) yields a central mass of $M=1.2 \times 10^{5} M_{\odot}$; if the calibrated reverberation mass scale of Onken et al. (2004) and Peterson et al. (2004) 
TABLE 6

Measured C iv $\lambda 1549$ Lags

\begin{tabular}{|c|c|c|c|c|}
\hline $\begin{array}{c}\text { Data Set } \\
\text { (1) }\end{array}$ & $\begin{array}{l}\text { Lag } \\
\text { (days) } \\
(2)\end{array}$ & $\begin{array}{c}\text { Reference } \\
\text { (3) }\end{array}$ & $\begin{array}{c}\log \lambda L_{\lambda}(\mathrm{UV}) \\
\left(\mathrm{ergs} \mathrm{s}^{-1}\right) \\
(4)\end{array}$ & $\begin{array}{c}\text { Reference } \\
\text { (5) }\end{array}$ \\
\hline \multicolumn{5}{|l|}{ NGC 4395: } \\
\hline Visit $2 \ldots \ldots \ldots \ldots \ldots$ & $0.033_{-0.013}^{+0.017}$ & 1 & $40.47 \pm 0.06$ & 1 \\
\hline Visit $3 \ldots \ldots \ldots \ldots . . . . . .$. & $0.046_{-0.013}^{+0.017}$ & 1 & $41.00 \pm 0.03$ & 1 \\
\hline NGC $3783 \ldots \ldots \ldots \ldots$ & $4.0_{-1.5}^{+1.0}$ & 2 & $43.59 \pm 0.09$ & 3 \\
\hline \multicolumn{5}{|l|}{ NGC 5548: } \\
\hline Year 1 ................ & $9.8_{-1.5}^{+1.9}$ & 2 & $43.66 \pm 0.14$ & 4 \\
\hline Year $5 \ldots \ldots \ldots \ldots . . . . .$. & $6.7_{-1.0}^{+0.9}$ & 2 & $43.58 \pm 0.06$ & 5 \\
\hline NGC $7469 \ldots \ldots \ldots . . . .$. & $2.5_{-0.2}^{+0.3}$ & 2 & $43.78 \pm 0.07$ & 6 \\
\hline 3C $390.3 \ldots \ldots \ldots \ldots . . . . .$. & $35.7_{-14.6}^{+11.4}$ & 2 & $44.07 \pm 0.21$ & 7 \\
\hline
\end{tabular}

References.-(1) This work; (2) Peterson et al. 2004; (3) Reichert et al. 1994; (4) Clavel et al. 1991; (5) Korista et al. 1995; (6) Wanders et al. 1997; (7) O'Brien et al. 1998.

is used, this value should be increased by about a factor of 1.8 to $M=2.1 \times 10^{5} M_{\odot}$. Filippenko \& Ho (2003) use the Kaspi et al. (2000) radius-luminosity relationship ( $(4.2)$ to estimate the size of the BLR, and they arrive at a mass estimate of $1.2 \times 10^{5} M_{\odot}$. Based on the break in the X-ray power density spectrum, Shih et al. (2003) estimate a black hole mass of $10^{4}$ $10^{5} M_{\odot}$. Newer data from Vaughn et al. (2005) lead to an estimate of $6 \times 10^{4} M$. On the basis of a probable detection of a 400 s quasi-periodic oscillation, Moran et al. (2005) place an upper limit to the mass of $9 \times 10^{5} M_{\odot}$. Our measured mass of $(3.6 \pm 1.1) \times 10^{5} M_{\odot}$ is generally consistent with these previous estimates.

\subsection{The Radius-Luminosity Relationship}

One of the goals of this project is to determine the slope of the BLR radius-luminosity relationship for the $\mathrm{C}$ IV-emitting region. We parameterize the relationship between the size of the line-emitting region $R_{\mathrm{BLR}}$ and the continuum luminosity $L$ as

$$
R_{\mathrm{BLR}}=\beta L^{\alpha}
$$

where $\alpha$ and $\beta$ are constants. Recent work on the size of the $\mathrm{H} \beta$-emitting region based on the compilation of reverberationmapping data from Peterson et al. (2004) yields $\alpha \approx 0.67 \pm 0.05$ using the optical continuum luminosity for $L$ and $\alpha \approx 0.56 \pm$ 0.05 when $L$ is the UV continuum luminosity (Kaspi et al. 2005). Whereas this relationship is fairly well characterized for $\mathrm{H} \beta$, it is still poorly known for $\mathrm{C}$ IV because of the limited number of UV monitoring programs that have been undertaken to date and because the few AGNs for which C IV response times have been successfully measured do not cover a wide range in luminosity. Since NGC 4395 is a factor of $\gtrsim 400$ less luminous than the other objects for which C IV lags have been measured, measurement of the $\mathrm{C}$ IV response time potentially provides a strong constraint on the slope $\alpha$.

In Table 6 we list all the reliably measured C IV response times from Peterson et al. (2004). The UV luminosities in Table 6 are computed from the observed fluxes during the monitoring campaigns that yielded the response times, corrected for reddening as described by Peterson et al., and converted to luminosities assuming a standard $\Lambda$ CDM cosmology with $H_{0}=70 \mathrm{~km} \mathrm{~s}^{-1}$ $\mathrm{Mpc}^{-1}, \Omega_{M}=0.3$, and $\Omega_{\Lambda}=0.7$ (Riess et al. 2004). In the case of NGC 4395, we use the UV fluxes from Table 3, assume Galactic extinction of $A_{B}=0.074 \mathrm{mag}$ (Schlegel et al. 1998), and adopt a distance $D=4.3 \mathrm{Mpc}$ (Thim et al. 2004) to obtain the

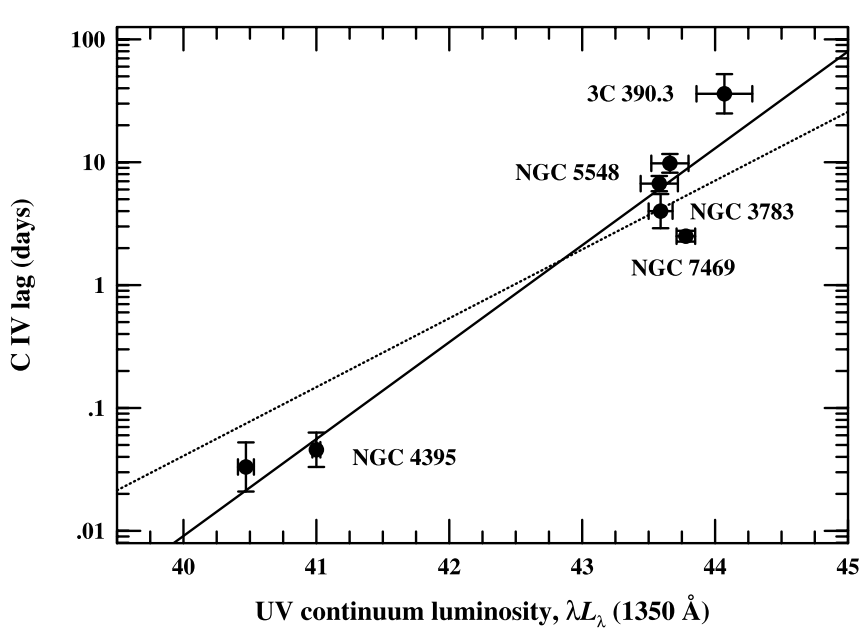

FIG. 6.-Radius-luminosity relationship based on the C IV $\lambda 1549$ emission line and the UV continuum, for the galaxies listed in Table 6. The UV continuum luminosity is in units of ergs $\mathrm{s}^{-1}$. The best-fit line, with slope $\alpha=$ $0.79 \pm 0.06$, is shown as a solid line. The dashed line is the best fit for a fixed slope $\alpha=0.56$, which is the slope of the relationship between the size of the $\mathrm{H} \beta$-emitting region and the UV luminosity (Kaspi et al. 2005).

UV luminosity. These values are plotted in Figure 6, along with the best-fit relationship

$$
\begin{aligned}
\log R_{\mathrm{BLR}}(\text { lt-days })=(1.11 \pm 0.16) \\
\quad+(0.79 \pm 0.06) \log \left(\frac{L_{\mathrm{UV}}}{10^{44} \mathrm{ergs} \mathrm{s}^{-1}}\right)
\end{aligned}
$$

The linear regression was performed using the BCES (bivariate correlated errors and intrinsic scatter) program described by Akritas \& Bershady (1996). This technique accounts for measurement errors in both parameters. The slope of this relationship $\alpha=0.79$ is fairly steep, a rather stronger dependence on the UV continuum luminosity than found for $\mathrm{H} \beta(\alpha=0.56)$, although admittedly over a very different range in luminosity. It remains unclear whether (1) the slope of the BLR radiusluminosity relationship is different in different luminosity ranges, (2) the slope of the radius-luminosity relationship is different for $\mathrm{C}$ IV and $\mathrm{H} \beta$, or (3) the NGC 4395 results are anomalous in some way. Certainly the most direct way to sort out these possibilities is to measure the $\mathrm{H} \beta$ response for NGC 4395 .

\subsection{The $M_{\mathrm{BH}}-\sigma_{*}$ Relationship}

One of the most extraordinary results of the last several years in the dynamics of galaxies has been the identification of a strong correlation between central black hole mass and the velocity dispersion of the stars in the host-galaxy bulge, the $M_{\mathrm{BH}}-\sigma_{*}$ relationship (Ferrarese \& Merritt 2000; Gebhardt et al. 2000a). This relationship was originally found in galaxies whose black hole masses were measured by modeling of stellar dynamics, gas dynamics, or megamaser motions, but it was subsequently shown to hold for sources with reverberation-based masses (Gebhardt et al. 2000b; Ferrarese et al. 2001; Onken et al. 2004; Nelson et al. 2004). Indeed, as described earlier, we can use the $M_{\mathrm{BH}} \sigma_{*}$ relationship to provide a zero-point calibration for the reverberation-based mass scale (Onken et al. 2004).

NGC 4395 is of special interest in this context as the host galaxy is essentially bulgeless and the central velocity dispersion has a measured upper limit of $\sigma_{*} \leq 30 \mathrm{~km} \mathrm{~s}^{-1}$ (Filippenko \& Ho 2003). Figure 7 shows a plot of the $M_{\mathrm{BH}^{-}} \sigma_{*}$ relationship including our new mass measurement for NGC 4395. NGC 4395 is something of an outlier in this relationship; indeed, the limit 


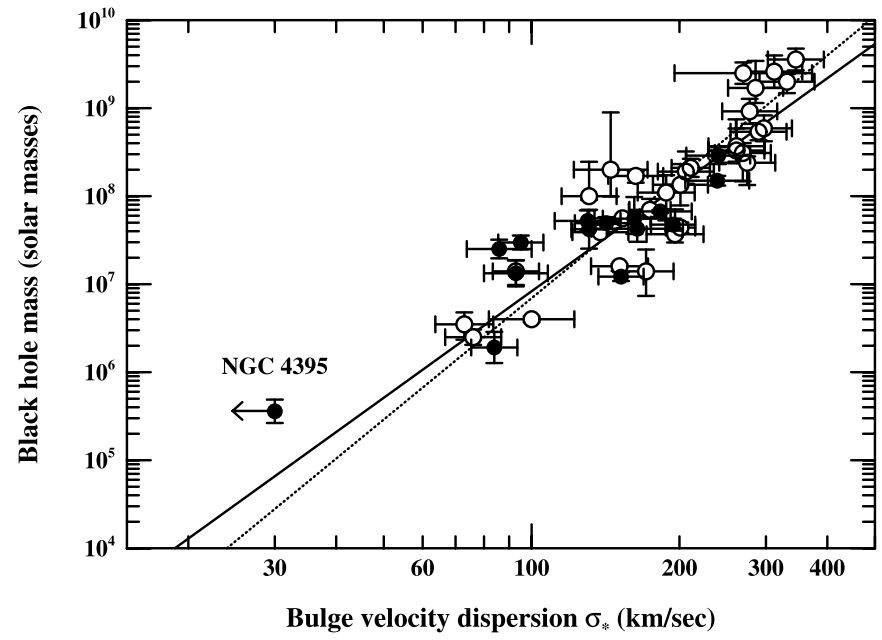

FIG. 7.- Relationship between black hole mass and stellar bulge velocity dispersion, the $M_{\mathrm{BH}}-\sigma_{*}$ relationship. Filled circles represent masses determined by reverberation mapping, including NGC 4395 in the lower left corner of the diagram; open circles are mass measurements by other techniques, as compiled by Tremaine et al. (2002). The solid line is the best fit to the non-reverberation measurements by Tremaine et al., and the dashed line is the best fit of Ferrarese (2002), based on a slightly different set of masses. The upper limit on the bulge velocity dispersion for NGC 4395, $\sigma_{*} \leq 30 \mathrm{~km} \mathrm{~s}^{-1}$, is from Filippenko \& Ho (2003).

$\sigma_{*} \leq 30 \mathrm{~km} \mathrm{~s}^{-1}$ implies that $M_{\mathrm{BH}} \leq 6.6 \times 10^{4} M_{\odot}$, more than a factor of 5 lower than our measured mass. Of course, the location of NGC 4395 in Figure 7 is not necessarily inconsistent with the intrinsic scatter in the $M_{\mathrm{BH}}-\sigma_{*}$ relationship as long as $\sigma_{*}$ is not far below the quoted upper limit; perhaps the more important point is that we find a supermassive black hole, albeit on the small side, in an essentially bulgeless galaxy for which the definition of $\sigma_{*}$ is in fact problematic.

Along the same lines, there is also a well-established empirical relationship between central black hole mass and bulge luminosity (Magorrian et al. 1998). Again, the problem with NGC 4395 is the apparent complete absence of a bulge component. However, if we take the central star cluster to be effectively the bulge component, then the bulge apparent magnitude is $I=16.8 \mathrm{mag}$ (Filippenko \& Ho 2003). Assuming that $B-I=1.57 \mathrm{mag}$, which is appropriate for the average stellar population of Scd galaxies (Fukugita et al. 1995), then $B=$ $18.37 \mathrm{mag}$ and $M_{B}=-9.75 \mathrm{mag}$. Using an updated calibration of the Magorrian relationship from Kormendy \& Gebhardt (2001), the expected black hole mass is $M_{\mathrm{BH}}=4.7 \times 10^{3} M_{\odot}$, a factor of about 80 smaller than our measured value.

\subsection{The AGN Mass-Luminosity Relationship}

Because of its extremely low luminosity, the location of NGC 4395 on the AGN mass-luminosity relationship is also of special interest, as seen in Figure 8. The value of the optical luminosity used here is based on our own ground-based observations (to be published elsewhere) that we obtained simultaneously with visit 2 . The mean optical flux for our observations is $\left\langle F_{\lambda}(5100 \AA)\right\rangle=(4.98 \pm 0.14) \times 10^{-16} \mathrm{ergs} \mathrm{s}^{-1} \mathrm{~cm}^{-2} \AA^{-1}$. We did not attempt to account for starlight contamination, which should be weak. As before, we assume Galactic extinction of $A_{B}=0.074 \mathrm{mag}$ and obtain $\lambda L_{\lambda}(5100 \AA)=5.9 \times 10^{39} \mathrm{ergs} \mathrm{s}^{-1}$, so that NGC 4395 is less luminous than any other reverberationmapped AGN by some 2 orders of magnitude.

The upper abscissa of Figure 8 shows the bolometric luminosity based on the very simple assumption that $L_{\text {bol }} \approx$ $9 \lambda L_{\lambda}(5100 \AA)$ (Kaspi et al. 2000). For NGC 4395, this implies

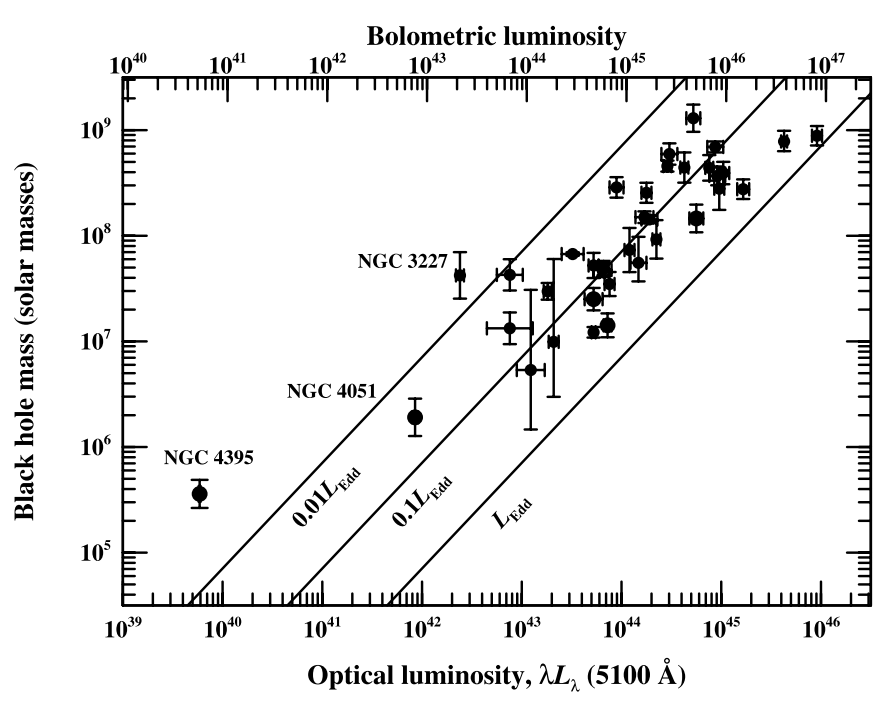

FIG. 8.- Relationship between black hole mass and AGN optical luminosity. The position of NGC 4395 is noted. Other data are from Peterson et al. (2004). The optical luminosity is in units of ergs $\mathrm{s}^{-1}$. The scale on the upper axis is bolometric luminosity, assuming that $L_{\mathrm{bol}} \approx 9 L_{\lambda}$. The diagonal lines show the Eddington limit $L_{\mathrm{Edd}}, 0.1 L_{\mathrm{Edd}}$, and $0.01 L_{\mathrm{Edd}}$. The only other source radiating below $1 \%$ of the Eddington rate is NGC 3227 , which is heavily absorbed and whose luminosity is therefore underestimated. However, both the Galactic and internal extinction are small in the case of NGC 4395, so absorption cannot account for its low Eddington rate, $\dot{m} \approx 0.0012$.

$L_{\mathrm{bol}} \approx 5.4 \times 10^{40} \mathrm{ergs} \mathrm{s}^{-1}$, which compares favorably with the estimate of Moran et al. (1999), $L_{\text {bol }} \approx(5.2-8.8) \times 10^{40} \mathrm{ergs} \mathrm{s}^{-1}$ (with the uncertainty depending on the internal extinction, which is not well constrained, although Moran et al. suggest an upper limit of $A_{V} \leq 0.2$ mag to avoid overcorrecting the $2175 \AA$ feature), adjusted to a distance $D=4.3 \mathrm{Mpc}$. Also shown in Figure 8 are loci of fixed Eddington ratio, i.e., $\dot{m}=\dot{M} / \dot{M}_{\text {Edd }}$. This plot clearly demonstrates that NGC 4395 is remarkable among reverberation-mapped AGNs in that its accretion rate is so low, $\dot{m} \approx 1.2 \times 10^{-3}$, a value more typical of LINERs and broad-line radio galaxies (Eracleous \& Halpern 2003) than Seyfert 1 galaxies. The only other source radiating at less than $1 \%$ of the Eddington rate is NGC 3227 , which is a heavily absorbed nucleus (Komossa \& Fink 1997; Crenshaw et al. 2001), whose intrinsic luminosity is thus underestimated. Certainly, however, one must be careful in drawing conclusions because the reverberation-mapped AGNs are not an unbiased sample; most of these sources were selected for reverberation study at least in part on account of their apparent brightness, which biases the sample toward higher values of $\dot{m}$, i.e., toward higher luminosity per unit mass.

\section{CONCLUSIONS}

We have successfully carried out a reverberation-mapping program on the least luminous known Seyfert 1 galaxy, NGC 4395, with HST STIS. The goals of this program were to measure (1) the size of the broad C IV-emitting region and (2) the mass of the central black hole. We reach the following conclusions:

1. Two independent visits with HST yielded measurements of the timescale for response of the broad C IV $\lambda 1549$ emission line to continuum variations of $\tau_{\text {cent }}=0.033_{-0.013}^{+0.017}$ and $0.046_{-0.013}^{+0.017}$ days for 2004 April and July, respectively, i.e., about $1 \mathrm{hr}$. The larger time lag measured in July is qualitatively consistent with the higher luminosity of the continuum at this time.

2. By combining the measured time lags with the line width of the variable part of the emission line, and using the zero-point 
normalization of the reverberation-based mass scale found by Onken et al. (2004), we obtain a central black hole mass of $M_{\mathrm{BH}}=(3.6 \pm 1.1) \times 10^{5} M_{\odot}$, where the uncertainty is the random component of the error due to uncertainties in the measurement of the emission-line lags and widths.

3. The size of the $\mathrm{C}$ IV-emitting region in NGC 4395 is somewhat smaller than expected based on similar measurements of the $\mathrm{C}$ iv response time in more luminous objects, assuming that the slope of the radius-luminosity relationship has the same slope for $\mathrm{C}$ IV and $\mathrm{H} \beta$. Either the slope of the radius-luminosity relationship is steeper at lower luminosities, or the slope is slightly steeper for $\mathrm{C}$ IV than for $\mathrm{H} \beta$, or our result for NGC 4395 is somewhat anomalous. For this reason, measuring the $\mathrm{H} \beta$ reverberation lag in NGC 4395 is important.

4. The existence of a supermassive black hole, albeit a small one, in a bulgeless galaxy demonstrates that a bulge is not a necessary condition for a nuclear black hole in a galaxy. This is an important point in light of the absence of a supermassive black hole in the bulgeless galaxy M33 (Gebhardt et al. 2001; Merritt et al. 2001). However, it may still be that a galactic bulge is a sufficient condition for the existence of a nuclear supermassive nuclear black hole.

5. In addition to having the smallest known supermassive black hole, NGC 4395 also has a very low accretion rate. Relative to other reverberation-mapped AGNs, it is remarkably underluminous for its mass. It was not obvious prior to this mass measurement whether the low luminosity of NGC 4395 was due to a smaller mass with a fairly typical Eddington rate or a larger mass with a low Eddington rate. This experiment argues for the latter.

We close by reminding the reader that the calibration of the reverberation-based mass scale is statistical; the scatter in the $M_{\mathrm{BH}^{-}} \sigma_{*}$ relationship indicates that the typical systematic un- certainties amount to about a factor of 3 . While apparently rare, it is possible for an individual mass estimate to be inaccurate by a larger factor. If indeed we have significantly overestimated the black hole mass of NGC 4395, the Eddington rate is similarly underestimated and the deviation from the $M_{\mathrm{BH}^{-}} \sigma_{*}$ relationship is correspondingly less marked.

We thank our program coordinator Tony Roman and contact scientist Linda Dressel at STScI for much help in the execution of this program. We are especially grateful to the STScI Telescope Time Review Board and Duccio Macchetto for approving our request for a repeat visit when visit 1 failed; without this, the results described here would be substantially less compelling. We are grateful for support of this research by NASA through grant HST-GO-09818 from the Space Telescope Science Institute, which is operated by the Association of Universities for Research in Astronomy, Inc., under NASA contract NAS526555, by the NSF through grant AST 02-05964 to the Ohio State University, by the Israel Science Foundation through grant 1030/04, and by a grant from the Norman and Helen Asher Space Research Institute. M. B. is supported by a graduate fellowship from the National Science Foundation. L.-B. D. is supported by a Julie-Payette Fellowship from the National Science and Engineering Research Council of Canada and the Canadian Space Agency. A. V. F. is grateful for a Miller Research Professorship at the University of California, Berkeley, during which part of this work was completed. S. K. is supported at the Technion in part by a Zeff Fellowship. This research has made use of the NASA/ IPAC Extragalactic Database (NED), which is operated by the Jet Propulsion Laboratory, California Institute of Technology, under contract with NASA.
Akritas, M. G., \& Bershady, M. A. 1996, ApJ, 470, 706

Alexander, T. 1997, in Astronomical Time Series, ed. D. Maoz, A. Sternberg, \& E. M. Leibowitz (Dordrecht: Kluwer), 163

Barth, A., Green, J. E., \& Ho, L. C. 2005, ApJ, 619, L151

Barth, A., Ho, L. C., Rutledge, R. E., \& Sargent, W. L. W. 2004, ApJ, 607, 90

Blandford, R. D., \& McKee, C. F. 1982, ApJ, 255, 419

Clavel, J., et al. 1991, ApJ, 366, 64

Crenshaw, D. M., Kraemer, S. B., Bruhweiler, F. C., \& Ruiz, J. R. 2001, ApJ, 555, 633

Dashevsky, I., Sahu, K., \& Smith, E. 2000, STIS Instrument Science Report 00-02 (Baltimore: STScI)

Edelson, R. A., \& Krolik, J. H. 1988, ApJ, 333, 646

Eracleous, M., \& Halpern, J. P. 2003, ApJ, 599, 886

Ferrarese, L. 2002, in Proc. 2nd KIAS Astrophysics Workshop, Current High-Energy Emission around Black Holes, ed. C.-H. Lee \& H.-Y. Chang (Singapore: World Scientific), 3

Ferrarese, L., \& Merritt, D. M. 2000, ApJ, 539, L9

Ferrarese, L., Pogge, R. W., Peterson, B. M., Merritt, D., Wandel, A., \& Joseph, C. L. 2001, ApJ, 555, L79

Filippenko, A. V., \& Ho, L. C. 2003, ApJ, 588, L13

Filippenko, A. V., Ho, L. C., \& Sargent, W. L. W. 1993, ApJ, 410, L75

Filippenko, A. V., \& Sargent, W. L. W. 1989, ApJ, 342, L11

Fukugita, M., Shimasaku, K., \& Ichikawa, T. 1995, PASP, 107, 945

Gaskell, C. M., \& Peterson, B. M. 1987, ApJS, 65, 1

Gaskell, C. M., \& Sparke, L. S. 1986, ApJ, 305, 175

Gebhardt, K., et al. 2000a, ApJ, 539, L13

$$
\text { . 2000b, ApJ, 543, L5 }
$$$$
\text { 2001, AJ, 122, } 2469
$$

Greene, J. E., \& Ho, L. C. 2004, ApJ, 610, 722

Häring, N., \& Rix, H.-W. 2004, ApJ, 604, L89

Ho, L. C., \& Peng, C. Y. 2001, ApJ, 555, 650

Ho, L. C., \& Ulvestad, J. S. 2001, ApJS, 133, 77

Ho, L. C., et al. 2001, ApJ, 549, L51

Iwasawa, K., Fabian, A. C., Almaini, O., Lira, P., Lawrence, A., Hayashida, K., \& Inoue, H. 2000, MNRAS, 318, 879
REFERENCES

Kaspi, S., Maoz, D., Netzer, H., Peterson, B. M., Vestergaard, M., \& Jannuzi, B. T. 2005, ApJ, 629, 61

Kaspi, S., Smith, P. S., Netzer, H., Maoz, D., Jannuzi, B. T., \& Giveon, U. 2000, ApJ, 533, 631

Keyes, T., ed. 1997, HST Data Handbook, Vol. 1, Current Instruments (Baltimore: STScI)

Komossa, S., \& Fink, H. 1997, A\&A, 327, 483

Korista, K. T., et al. 1995, ApJS, 97, 285

Kormendy, J., \& Gebhardt, K. 2001, in AIP Conf. Proc. 586, 20th Texas Symposium on Relativistic Astrophysics, ed. H. Martel \& J. C. Wheeler (Melville: AIP), 363

Kraemer, S., Ho, L. C., Crenshaw, D. M., Shields, J. C., \& Filippenko, A. V. 1999, ApJ, 520, 564

Laor, A. 1998, ApJ, 505, L83

Lira, P., Lawrence, A., O’Brien, P., Johnson, R. A., Terlevich, R., \& Bannister, N. 1999, MNRAS, 305, 109

Magorrian, J., et al. 1998, AJ, 115, 2285

Marconi, A., \& Hunt, L. K. 2003, ApJ, 589, L21

McLure, R. J., \& Jarvis, M. J. 2002, MNRAS, 337, 109

Merritt, D., Ferrarese, L., \& Joseph, C. L. 2001, Science, 293, 1116

Moran, E. C., Eracleous, M., Leighly, K. M., Chartas, G., Filippenko, A. V., Ho, L. C., \& Blanco, P. R. 2005, AJ, 129, 2108

Moran, E. C., Filippenko, A. V., Ho, L. C., Shields, J. C., Belloni, T., Comastri, A., Snowden, S. L., \& Sramek, R. A. 1999, PASP, 111, 801

Nelson, C. H., Green, R. F., Bower, G., Gebhardt, K., \& Weistrop, D. 2004, ApJ, 615, 652

Netzer, H., \& Peterson, B. M. 1997, in Astronomical Time Series, ed. D. Maoz, A. Sternberg, \& E. M. Leibowitz (Dordrecht: Kluwer), 85

O’Brien, P. T., et al. 1998, ApJ, 509, 163

Onken, C. A., Ferrarese, L., Merritt, D., Peterson, B. M., Pogge, R. W., Vestergaard, M., \& Wandel, A. 2004, ApJ, 615, 645

Peterson, B. M. 1993, PASP, 105, 247

. 2001, in Advanced Lectures on the Starburst-AGN Connection, ed. I. Aretxaga, D. Kunth, \& R. Mújica (Singapore: World Scientific), 3 
Peterson, B. M., Wanders, I., Horne, K., Collier, S., Alexander, T., \& Kaspi, S. 1998, PASP, 110, 660

Peterson, B. M., et al. 2002, ApJ, 581, 197 2004, ApJ, 613, 682

Reichert, G. A., et al. 1994, ApJ, 425, 582

Riess, A. G., et al. 2004, ApJ, 607, 665

Rodríguez-Pascual, P. M., et al. 1997, ApJS, 110, 9

Schlegel, D. J., Finkbeiner, D. P., \& Davis, M. 1998, ApJ, 500, 525

Seyfert, C. K. 1943, ApJ, 97, 28

Shih, D. C., Iwasawa, K., \& Fabian, A. C. 2003, MNRAS, 341, 973

Skelton, J. E., Lawrence, A., Pappa, A., Lira, P., \& Almaini, O. 2005, MNRAS, 358,781
Thim, F., Hoessel, J. G., Saha, A., Claver, J., Dolphin, A., \& Tammann, G. A. 2004, AJ, 127, 2322

Tremaine, S., et al. 2002, ApJ, 574, 740

Vaughan, S., Iwasawa, K., Fabian, A. C., \& Hayashida, K. 2005, MNRAS, 356, 524

Vestergaard, M. 2002, ApJ, 571, 733

Wanders, I., et al. 1997, ApJS, 113, 69

White, R. J., \& Peterson, B. M. 1994, PASP, 106, 879

Wrobel, J. M., Fassnacht, C. D., \& Ho, L. C. 2001, ApJ, 553, L23 


\title{
ERRATUM: "MULTIWAVELENGTH MONITORING OF THE DWARF SEYFERT 1 GALAXY NGC 4395. I. A REVERBERATION-BASED MEASUREMENT OF THE BLACK HOLE MASS” (ApJ, 632, 799 [2005])
}

\author{
Bradley M. Peterson, Misty C. Bentz, louis-Benoit Desroches, Alexei V. Filippenko, Luis C. Ho, Shai Kaspi, \\ Ari Laor, Dan Maoz, Edward C. Moran, Richard W. Pogge, and Alice C. Quillen
}

In the original version of this paper, we reported on a series of ultraviolet (UV) spectroscopic observations of the dwarf Seyfert 1 galaxy NGC 4395, made with the Space Telescope Imaging Spectrograph on the Hubble Space Telescope (HST). Unfortunately, a data processing error led to an incorrect flux calibration for these spectra. All STIS-based UV fluxes in the original paper are too high by a factor of 7.96 as a result of neglecting to adjust the flux-scaling algorithm from a diffuse source to a point source.

Because most of the analysis in the original paper involved only relative flux changes, most of the scientific conclusions are unaffected by this correction, except for the slope of the broad-line region radius-luminosity relationship, as described below.

Specific changes that result from this correction are:

1. All of the values for the continuum and $\mathrm{C}$ IV emission-line fluxes and their associated uncertainties in the electronic Tables 1 and 2 need to be multiplied by a factor of 0.126 .

2. The mean and rms fluxes in column (3) of Table 3 need to be multiplied by a factor of 0.126 .

3 . The luminosity entries for NGC 4395 in column (4) of Table 6 need to be multiplied by a factor of 0.126 . A corrected version of Table 6 is provided here.

4. The flux scales in Figs. 1, 2, and 5 should be multiplied by a factor of 0.126 .

The only substantive change resulting from this correction is the relationship between the broad-line region radius, as measured by the time response of the $\mathrm{C}$ IV emission line, and the UV continuum luminosity. We show here the corrected version of Figure 6, based on the entries in the corrected version of Table 6 . The best-fit power-law relationship to these data is

$$
\log R_{\mathrm{BLR}}(\text { lt-days })=(1.06 \pm 0.16)+(0.61 \pm 0.05) \log \left(\frac{L_{\mathrm{UV}}}{10^{44} \operatorname{ergs~s}^{-1}}\right)
$$

which replaces equation (3) in the original paper. The slope $\alpha=0.61 \pm 0.05$ is in much better agreement with the slope of the radiusluminosity relationship of S. Kaspi et al. (ApJ, 629, 61 [2005]) for $\mathrm{H} \beta$ and the UV continuum, $\alpha=0.56 \pm 0.05$.

TABLE 6

Measured C iv $\lambda 1549$ Lags

\begin{tabular}{|c|c|c|c|c|}
\hline $\begin{array}{c}\text { Data Set } \\
\text { (1) }\end{array}$ & $\begin{array}{l}\text { Lag } \\
\text { (days) } \\
(2)\end{array}$ & $\begin{array}{c}\text { Reference } \\
\text { (3) }\end{array}$ & $\begin{array}{c}\log \lambda L_{\lambda}(\mathrm{UV}) \\
\left(\operatorname{ergs~s}^{-1}\right) \\
(4)\end{array}$ & $\begin{array}{c}\text { Reference } \\
\text { (5) }\end{array}$ \\
\hline \multicolumn{5}{|l|}{ NGC 4395: } \\
\hline Visit 2 & $0.033_{-0.013}^{+0.017}$ & 1 & $39.57 \pm 0.06$ & 1 \\
\hline Visit $3 \ldots \ldots \ldots \ldots \ldots \ldots \ldots \ldots \ldots \ldots \ldots \ldots$ & $0.046_{-0.013}^{+0.017}$ & 1 & $40.10 \pm 0.03$ & 1 \\
\hline 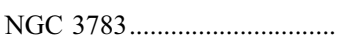 & $4.0_{-1.5}^{+1.0}$ & 2 & $43.59 \pm 0.09$ & 3 \\
\hline \multicolumn{5}{|l|}{ NGC 5548: } \\
\hline . & $9.8_{-15}^{+1.9}$ & 2 & $43.66 \pm 0.14$ & 4 \\
\hline 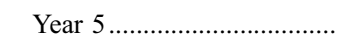 & $6.7_{-1.0}^{+0.9}$ & 2 & $43.58 \pm 0.06$ & 5 \\
\hline 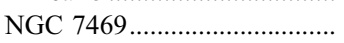 & $2.5_{-0.2}^{+0.0}$ & 2 & $43.78 \pm 0.07$ & 6 \\
\hline 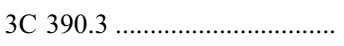 & $35.7_{-14.6}^{+11.4}$ & 2 & $44.07 \pm 0.21$ & 7 \\
\hline
\end{tabular}

References.-(1) This work; (2) Peterson et al. 2004; (3) Reichert et al. 1994; (4) Clavel et al. 1991; (5) Korista et al. 1995; (6) Wanders et al. 1997; (7) O’Brien et al. 1998. 


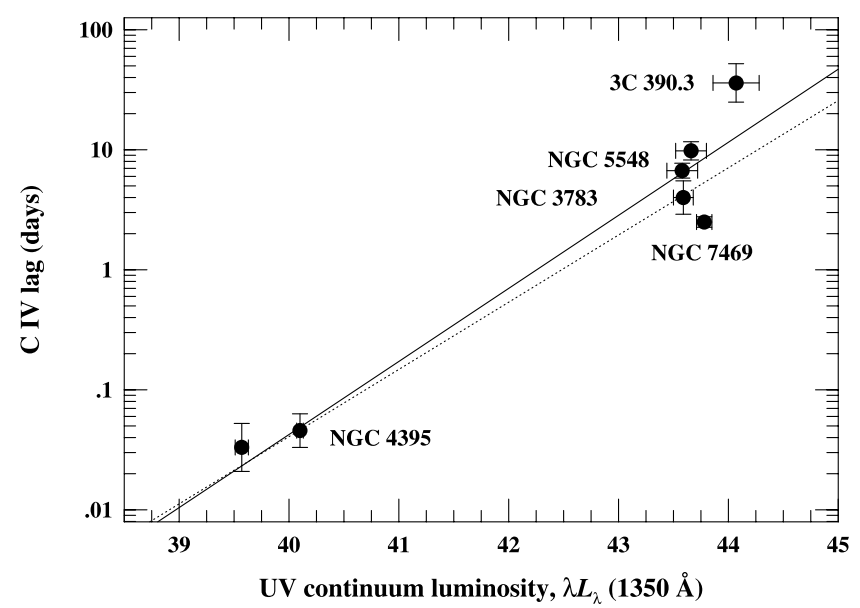

FIg. 6.-Radius-luminosity relationship based on the $\mathrm{C}$ iv $\lambda 1549$ emission line and the UV continuum, for the galaxies listed in Table 6 . The UV continuum luminosity is in units of ergs s ${ }^{-1}$. The best-fit line, with slope $\alpha=0.61 \pm 0.05$, is shown as a solid line. The dashed line is the best fit for a fixed slope $\alpha=0.56$, which is the slope of the relationship between the size of the $\mathrm{H} \beta$-emitting region and the UV luminosity (Kaspi et al. 2005). 